\title{
The presence of perceived stress among primary and non- primary adult child caregivers in the Greater Chicago Area
}

\author{
Mengting $\mathrm{LI}^{1}$ and Xinqi Dong* ${ }^{* 2}$ \\ ${ }^{1}$ Post-Doctoral Research Fellow, Rush Institute for Healthy Aging, Rush University Medical Center, USA \\ ${ }^{2}$ Professor of Medicine, Nursing, and Behavioral Sciences, Rush Institute for Healthy Aging, USA
}

\begin{abstract}
Background: A wealthy of literature constructed caregiving as a stressful event. This study aims to examine the association between caregiver role and perceived stress by comparing the perceived stress of primary and non-primary adult child caregivers.

Methods: Data were derived from the Piety Study, a community-engaged study of U.S. Chinese adult children who were 21 years and older living in the greater Chicago area. Perceived stress was evaluated by the Chinese Perceived Stress Scale. Linear regression analyses were performed.

Results: Primary adult child caregivers were more likely to be employed $\left(\chi^{2}=9.00, p<.01\right)$ and have higher income $\left(\chi^{2}=11.46, p<.01\right)$ than non-primary adult child caregivers. The overall health status between primary adult child caregivers and non-primary adult child caregivers was not significant $\left(\chi^{2}=3.15\right.$, $\left.p=.37\right)$. The caregiver role was not significantly associated with perceived stress (Parameter Estimate $(\mathrm{PE})=-.427$, Standard Error $(\mathrm{SE})=.587, p=.47)$.

Discussion: Healthy caregiver hypothesis was supported in this study while we also proposed wealthy caregiver hypothesis. As the number of ageing population largely increased in the past decades, a more balanced and updated profile of family caregivers for older adults is needed to encourage more adult children to assume primary caregiver roles.
\end{abstract}

\section{Introduction}

The stress process model served as the major orientating framework for understanding the consequences of family caregiving in the past decades [1]. The stress process theory constructs family caregiving as a stressful event and family caregivers are associated with negative health outcomes. However, recent studies found better health outcomes in primary caregivers than non-primary caregivers. The healthy caregiver hypothesis has been proposed as an alternative paradigm to explain positive caregiving consequences.

The healthy caregiver hypothesis suggests that old persons who are healthier assume primary caregiver role, leading to better health outcomes in primary caregivers than non-primary caregivers of the same cohort. This hypothesis was supported by studies that found older adults who took over primary caregiver role were physically healthier, and had lower rates of functional decline, cognitive decline and mortality than non-primary caregivers [2-7].

Previous research tested the healthy caregiver hypothesis in old caregivers sample and most of them were spouse caregivers. However, healthy caregiver hypothesis has rarely been tested in adult child caregivers. Adult children constitute the largest group of primary caregivers around the globe. For example, $42 \%$ of primary caregivers were adult child caregivers in the United States [8]. Particularly, the proportion of Chinese immigrants in the U.S. who assume primary caregiver role for ageing parents may be overwhelmingly high due to its relevance in Chinese culture.

Whether U.S. Chinese adult child caregiver would follow the stress process model or healthy caregiver hypothesis remains unclear. Primary adult child caregiver may experience high stress during caregiving process as they regard providing care for ageing parents as extra work in their daily life $[9,10]$. Some research indicated the collectivistic cultural values such as familism would attenuate stress appraisal [11]. In Chinese culture, adult children are more likely to regard caregiving as a normal part of daily life rather than sacrifice [12]. However, the stress experienced in taking care of ageing parents for U.S. Chinese adult children immigrants may be different from those who stay in China. Cultural values may shift with immigration to the U.S. [13,14]. Adult children are generally able to adapt easier to Western culture and may endorse such western ideologies as individualism.

Early studies on family caregiving have largely utilized samples composed exclusively of primary caregivers, which makes it difficult to identify whether high levels of stress was emanating from caregiving $[15,16]$. Research on both primary caregivers and non-primary caregivers can demonstrate more clearly how informal caregiving experiences affect the perceived stress of primary caregivers.

In this study, we aim to: 1) describe the prevalence of perceived stress in a community-dwelling population of U.S. Chinese adult children; 2) examine the reliability of the Chinese Perceived Stress Scale

Correspondence to: Xinqi Dong, MD, MPH, Professor of Medicine, Nursing, and Behavioral Sciences, Director, Chinese Health, Aging and Policy Program, Associate Director, Rush Institute for Healthy Aging Rush University Medical Center, USA, Tel: No: 312942 3350; Fax: 312942 2861; E-mail: xinqi_dong@ rush.edu

Key words: perceived stress, adult child caregiver, primary caregiver, Chinese

Received: February 12, 2017; Accepted: March 15, 2017; Published: March 18, 2017 
among U.S. Chinese adult children; and 3) investigate the association between primary caregiver role and the perceived stress among U.S. Chinese adult children.

\section{Methods}

\section{Sample}

The Piety Study is a community-engaged study of U.S. Chinese adult children living in the greater Chicago area. Adult children aged 21 years and older, and having at least one parent who is Chinese aged 60 years and older were eligible to participate in the study. Primary adult child caregivers were defined as the adult children who assuming the primary caregiver role for mother or father. Non-primary adult child caregivers referred to those who were neither the primary caregiver for their mother nor for their father. The sample size was 548 . Face-to-face home interviews were conducted by trained multicultural and multilingual interviewers. Preferred language (English or Chinese) and dialect (e.g. Cantonese, Taishanese, Mandarin, and Teochew) for participants were used during the interview.

\section{Measurements}

Sociodemographics: Basic demographic information collected included age, sex, education, annual personal income, marital status, employment status, quality of life and general health. Education was measured by the years of education completed. Self-reported annual income was divided into four groups: 1) $\$ 0-\$ 4,999$ per year; 2$) \$ 5,000$ $\$ 9,999$ per year; 3 ) $\$ 10,000-14,999$ per year; and 4) more than $\$ 15,000$ per year. Overall health status was assessed by self-reporting, based on the four-point scale ( 1 = very good, 2 = good, 3 = fair, 4 = poor). Quality of life was assessed by asking "In general, how would you rate your quality of life?" and the answer was based on a four-point scale $(1=$ poor, 2 = fair, 3 = good, 4 = very good).

Perceived stress: Individual's appraisal of a situation as stressful is regarded as the best indicator of the experience of stress [17]. Perceived Stress Scale was widely used to evaluate the degree to which participants perceived their lives as being unpredictable, uncontrollable, and overloading $[18,19]$. In this study, the Chinese Perceived Stress Scale (PSS-10) was adopted to evaluate the degree of perceived stress among Chinese adult children caregivers. Respondents indicated their answers using a 5 -point scale $(0=$ never, $1=$ almost never, 2 = fairly often, 3 = fairly often, $4=$ very often). After recoding the items in the same direction, we added up the ten items, with higher scores indicating higher perceived stress. Content validity of PSS-10 has been examined by previous research. A study in the U.S. revealed internal reliability score (Cronbach's alpha) on the PSS-10 was .88 among Alzheimer's disease caregivers [15]. The PINE study reported internal reliability score for the PSS-10 was .86 among U.S. Chinese older adults [20].

\section{Data analysis}

Descriptive analyses were used to describe the general sociodemographic characteristics of primary and non-primary adult child caregivers. Chi-squared tests were used to evaluate socio-demographic differences between participants. Linear regression models were used to examine whether perceived stress levels were associated with age, sex, education, annual personal income, marital status, employment, quality of life, general health and primary caregiver role. Statistical analyses were conducted using SAS, V9.2 (SAS Institute Inc., Cary, NC).

\section{Results}

\section{Characteristics of the study sample}

In the present study sample, 393 were primary adult child caregivers for their ageing parents and 149 were non-primary adult child caregivers (Table 1). With regard to primary adult child caregivers, the majority of them were female $(64.89 \%)$, married $(79.90 \%)$ and employed (86.01\%). Most of them aged 35 to 49 (51.15\%), with an education level between 7 to 12 years (55.22\%), reported good quality of life (53.18\%) and good overall health status (51.65\%), and had an annual income between $\$ 5,000$ to $\$ 9,999$ (34.87\%).

As for non-primary adult child caregivers, the majority of them were female (65.77\%), married (85.23\%) and employed (75.17\%). The largest proportion of them were between 35 to 49 (50.34\%), had an education level between 7 to 12 years $(62.42 \%)$, reported good quality of life (55.03\%) and good overall health status (48.99\%), and had an annual income between $\$ 0$ to $\$ 4,999$ (36.91\%).

Table 1. Demographic characteristics of adult children by primary caregiver role.

\begin{tabular}{|c|c|c|c|c|}
\hline & $\begin{array}{c}\text { Primary } \\
\text { Caregiver } \\
(\mathbf{n}=\mathbf{3 9 3})\end{array}$ & $\begin{array}{c}\text { Non-Primary } \\
\text { Caregiver } \\
(n=149)\end{array}$ & $\chi^{2}$ & $p$ value \\
\hline Age & & & \multirow{5}{*}{7.80} & \multirow{5}{*}{.05} \\
\hline $21-34$ & 11.96 & 4.70 & & \\
\hline $35-49$ & 51.15 & 50.34 & & \\
\hline $50-64$ & 31.55 & 39.60 & & \\
\hline $65-79$ & 5.34 & 5.37 & & \\
\hline Sex & & & \multirow{3}{*}{.04} & \multirow{3}{*}{.85} \\
\hline Male & 35.11 & 34.23 & & \\
\hline Female & 64.89 & 65.77 & & \\
\hline $\begin{array}{c}\text { Income in US \$, } \\
\text { numbers }(\%)\end{array}$ & & & \multirow{5}{*}{11.46} & \multirow{5}{*}{$<.01$} \\
\hline $0-4,999$ & 23.85 & 36.91 & & \\
\hline $5,000-9,999$ & 34.87 & 33.56 & & \\
\hline $10,000-14,999$ & 18.21 & 15.44 & & \\
\hline $\begin{array}{l}15,000 \text { and } \\
\text { more }\end{array}$ & 23.08 & 14.09 & & \\
\hline \multicolumn{3}{|l|}{ Marital status } & \multirow{3}{*}{2.03} & \multirow{3}{*}{.15} \\
\hline Married & 79.90 & 85.23 & & \\
\hline Other status & 20.10 & 14.77 & & \\
\hline \multicolumn{3}{|l|}{$\begin{array}{c}\text { Education level, } \\
\text { numbers }(\%)\end{array}$} & \multirow{6}{*}{12.52} & \multirow{6}{*}{$<.05$} \\
\hline 0 year & .25 & 0 & & \\
\hline 1-6 years & 6.87 & 12.75 & & \\
\hline $7-12$ years & 55.22 & 62.42 & & \\
\hline $13-16$ years & 26.21 & 20.13 & & \\
\hline 17 and above & 11.45 & 4.70 & & \\
\hline \multicolumn{3}{|l|}{$\begin{array}{l}\text { Employment } \\
\text { status }\end{array}$} & \multirow{3}{*}{9.00} & \multirow{3}{*}{$<.01$} \\
\hline Employed & 86.01 & 75.17 & & \\
\hline Unemployed & 13.99 & 24.83 & & \\
\hline \multicolumn{3}{|l|}{ Quality of life } & \multirow{5}{*}{.16} & \multirow{5}{*}{.98} \\
\hline Very good & 7.89 & 7.38 & & \\
\hline Good & 53.18 & 55.03 & & \\
\hline Fair & 36.13 & 34.90 & & \\
\hline Poor & 2.80 & 2.68 & & \\
\hline \multicolumn{3}{|l|}{$\begin{array}{l}\text { Overall health } \\
\text { status }\end{array}$} & \multirow{5}{*}{3.15} & \multirow{5}{*}{.37} \\
\hline Very good & 9.16 & 6.04 & & \\
\hline Good & 51.65 & 48.99 & & \\
\hline Fair & 33.33 & 40.27 & & \\
\hline Poor & 5.85 & 4.70 & & \\
\hline
\end{tabular}


Chi square test was conducted for comparing the differences of demographic factors between primary and non-primary adult child caregivers. The results showed education $\left(\chi^{2}=12.52, p<.05\right)$, employment status $\left(\chi^{2}=9.00, p<.01\right)$ and income $\left(\chi^{2}=11.46, p<\right.$ $.01)$ were significantly different between groups. Primary adult child caregivers were more likely to be employed and have higher income than their counterparts.

\section{Scale reliability}

In our sample, the alpha coefficient of reliability for PSS-10 was .83. The ten items in the scale were significantly correlated (Table 2). However, item 7 that being able to control irritations in life showed a weaker correlation with other items in the scale. If we delete item 7 , the alpha would slightly increase to .84. Compared with other items in PSS10 , upset because something happened unexpectedly and unable to control important things had higher inter-item correlation coefficients.

\section{Prevalence of perceived stress by primary and non-primary adult child caregivers}

Table 3 shows the prevalence of perceived stress by primary caregiver and non-primary caregiver. Among primary adult child caregivers, $92.88 \%$ of them have experienced some level of stress. The largest proportion of participants (33.34\%) sometimes, fairly often, or often felt they could not cope with all the things had to. Of the four items worded in a positive direction, never or almost never able to control irritations in life was reported by the largest number of primary adult child caregivers (12.47\%).
Among non-primary adult child caregivers, $94.63 \%$ perceived some form of stress. The largest number of non-primary adult child caregiver (30.88\%) sometimes, fairly often, or often felt nervous and stressed. Of the four items worded in a positive direction, never or almost never able to control irritations in life was most commonly reported by nonprimary adult child caregivers (13.42\%).

\section{Association between primary caregiver role and perceived stress}

The association between the primary caregiver role and perceived stress is presented in Table 4. The caregiver role was not significantly associated with perceived stress $(\mathrm{PE}=-.427, \mathrm{SE}=.587, \mathrm{p}=.47)$. In the fully adjusted model (Model $\mathrm{C})$, age $(\mathrm{PE}=-.068, \mathrm{SE}=.028, p<.05)$, sex $(\mathrm{PE}=1.517, \mathrm{SE}=.547, p<.01)$, education $(\mathrm{PE}=.295, \mathrm{SE}=.084$, $p<.001)$ and general health $(\mathrm{PE}=2.084, \mathrm{SE}=.378, p<.001)$ were significantly associated with perceived stress of adult children.

\section{Discussion}

Overall, $93.04 \%$ of adult children have perceived some form of stress in their daily lives. Adult children who were younger, female, had higher education and poorer health status were more likely to perceive higher levels of stress. As for primary caregivers, we found that $92.88 \%$ have experienced some level of stress in their daily lives. With regard to non-primary caregivers, $94.63 \%$ perceived some form of stress in their daily lives. However, the caregiver role was not significantly associated with perceived stress. Employment status and income were significantly different between primary and non-primary adult child caregivers.

Table 2. Perceived stress scale item correlation coefficients.

\begin{tabular}{|c|c|c|c|c|c|c|c|c|c|c|c|}
\hline Perceived Stress Scale Items & $\begin{array}{l}\text { Alpha } \\
\text { if item } \\
\text { deleted }\end{array}$ & 1 & 2 & 3 & 4 & 5 & 6 & 7 & 8 & 9 & 10 \\
\hline 1.Upset because something happened unexpectedly & 0.8 & 1 & & & & & & & & & \\
\hline 2.Felt unable to control important things & 0.8 & $.56^{* * *}$ & 1 & & & & & & & & \\
\hline 3.Felt nervous and stressed & 0.8 & $.55^{* * *}$ & $.53^{* * *}$ & 1 & & & & & & & \\
\hline 4.Confident about the ability to handle personal problems & 0.82 & $.26^{* * *}$ & $.26^{* * *}$ & $.27^{* * * *}$ & 1 & & & & & & \\
\hline 5.Felt things were going your way & 0.82 & $.29^{* * *}$ & $.27^{* * *}$ & $.29^{* * *}$ & $.40^{* * *}$ & 1 & & & & & \\
\hline 6.Could not cope with all the things had to & 0.81 & $.38^{* * *}$ & $.45^{* * *}$ & $.40^{* * * *}$ & $.26^{* * *}$ & $.23^{* * *}$ & 1 & & & & \\
\hline 7.Been able to control irritations in life & 0.84 & $.15^{* * *}$ & $.12^{* *}$ & $.13^{* *}$ & $.19^{* * * *}$ & $.18^{* * * *}$ & $.16^{* * * *}$ & 1 & & & \\
\hline 8.Felt you were on top of things & 0.81 & $.31^{* * *}$ & $.27^{* * *}$ & $.31^{* * *}$ & $.40^{* * *}$ & $.44^{* * * *}$ & $.27^{* * *}$ & $.31^{* * * *}$ & 1 & & \\
\hline 9.Angered because things happened out of control & 0.81 & $.42^{* * *}$ & $.40^{* * * *}$ & $.44^{* * * *}$ & $.28^{* * * *}$ & $.28^{* * * *}$ & $.44^{* * *}$ & $.25^{* * * *}$ & $.30^{* * * *}$ & 1 & \\
\hline 10. Felt could not overcome piled up difficulties & 0.8 & $.45^{* * *}$ & $.49^{* * *}$ & $.46^{* * * *}$ & $.31^{* * * *}$ & $.28^{* * * *}$ & $.45^{* * *}$ & $.26^{* * * *}$ & $.32^{* * * *}$ & $.52^{* * *}$ & 1 \\
\hline
\end{tabular}

Note. ${ }^{*} \mathrm{p}<.05,{ }^{* * *} \mathrm{p}<.01,{ }^{* * * *} \mathrm{p}<.001$

Table 3. Presence of perceived stress by primary caregiver and non-primary caregiver

\begin{tabular}{|c|c|c|c|c|c|c|c|c|c|c|}
\hline & \multicolumn{2}{|c|}{ Never $(\%)$} & \multicolumn{2}{|c|}{ Almost never (\%) } & \multicolumn{2}{|c|}{ Sometimes (\%) } & \multicolumn{2}{|c|}{ Fairly often (\%) } & \multicolumn{2}{|c|}{ Very often (\%) } \\
\hline & $\begin{array}{l}\text { Primary } \\
\text { Caregiver }\end{array}$ & $\begin{array}{c}\text { Non-Primary } \\
\text { Caregiver }\end{array}$ & $\begin{array}{l}\text { Primary } \\
\text { Caregiver }\end{array}$ & $\begin{array}{c}\text { Non-Primary } \\
\text { Caregiver }\end{array}$ & $\begin{array}{l}\text { Primary } \\
\text { Caregiver }\end{array}$ & $\begin{array}{c}\text { Non-Primary } \\
\text { Caregiver }\end{array}$ & $\begin{array}{l}\text { Primary } \\
\text { Caregiver }\end{array}$ & $\begin{array}{c}\text { Non-Primary } \\
\text { Caregiver }\end{array}$ & $\begin{array}{l}\text { Primary } \\
\text { Caregiver }\end{array}$ & $\begin{array}{l}\text { Non-Primary } \\
\text { Caregiver }\end{array}$ \\
\hline $\begin{array}{l}\text { 1.Upset because something happened } \\
\text { unexpectedly }\end{array}$ & 61.32 & 61.07 & 13.74 & 17.45 & 16.54 & 16.11 & 6.36 & 4.70 & 2.04 & 0.67 \\
\hline 2.Felt unable to control important things & 64.80 & 61.74 & 16.07 & 12.75 & 13.01 & 14.09 & 5.10 & 9.40 & 1.02 & 2.01 \\
\hline 3.Felt nervous and stressed & 50.64 & 51.01 & 17.30 & 18.12 & 21.12 & 20.81 & 9.16 & 6.04 & 1.78 & 4.03 \\
\hline $\begin{array}{l}\text { 4.Confident about the ability to handle } \\
\text { personal problems }\end{array}$ & 1.78 & 2.01 & 2.54 & 5.37 & 12.47 & 16.11 & 41.73 & 45.64 & 41.48 & 30.87 \\
\hline 5.Felt things were going your way & 5.34 & 1.34 & 5.34 & 9.40 & 27.48 & 24.16 & 37.40 & 40.94 & 24.43 & 24.16 \\
\hline 6.Could not cope with all the things had to & 48.35 & 42.95 & 18.32 & 28.19 & 25.70 & 20.81 & 5.60 & 5.37 & 2.04 & 2.68 \\
\hline 7. Been able to control irritations in life & 5.85 & 6.04 & 6.62 & 7.38 & 16.79 & 12.08 & 36.64 & 43.62 & 34.10 & 30.87 \\
\hline 8.Felt you were on top of things & 3.06 & 4.70 & 6.63 & 7.38 & 23.72 & 31.54 & 41.84 & 38.26 & 24.74 & 18.12 \\
\hline $\begin{array}{l}\text { 9.Angered because things happened out of } \\
\text { control }\end{array}$ & 52.93 & 53.69 & 22.39 & 22.82 & 20.87 & 19.46 & 3.05 & 2.68 & .76 & 1.34 \\
\hline $\begin{array}{l}\text { 10. Felt could not overcome piled up } \\
\text { difficulties }\end{array}$ & 64.89 & 63.76 & 22.39 & 25.50 & 9.92 & 8.05 & 2.29 & 1.34 & .51 & 1.34 \\
\hline
\end{tabular}


Table 4. Perceived stress regression model estimated parameter.

\begin{tabular}{|c|c|c|c|}
\hline & Model A & Model B & Model C \\
\hline \multicolumn{4}{|c|}{$\mathrm{b}(\mathrm{SE})$} \\
\hline Age & $-.047(.026)$ & $-.045(.029)$ & $-.068(.028)^{*}$ \\
\hline Female & $1.390(.566)^{*}$ & $1.587(.564)^{* * *}$ & $1.517(.547)^{* * *}$ \\
\hline Income & & $-.276(.131)^{*}$ & $-.249(.128)$ \\
\hline Married & & $-.863(.693)$ & $-.678(.673)$ \\
\hline Education & & $.252(.085)^{* * *}$ & $.295(.084)^{* * * *}$ \\
\hline Employed & & $-1.884(.837)^{*}$ & $-1.431(.815)$ \\
\hline Quality of life & & & $-.708(.398)$ \\
\hline General health & & & $2.084(.378)^{* * * *}$ \\
\hline Primary caregiver role & $-.541(.607)$ & $-.410(.606)$ & $-.427(.587)$ \\
\hline
\end{tabular}

Note. ${ }^{*} \mathrm{p}<.05,{ }^{* * *} \mathrm{p}<.01,{ }^{* * * *} \mathrm{p}<.001$

Consistent with previous research, our study found adult children who are younger, female, with higher education and poorer health status were more likely to perceive higher levels of stress $[21,22]$. Whereas, in the corresponding PINE study, which also used PSS-10 to examine the perceived stress among U.S. Chinese older adults, respondents with an older age were more likely to report higher levels of stress [20,23]. This may be explained that as for older adults, old age was associated with deterioration in health and thus resulted in perceiving higher levels of stress. But for adult children, they have competing roles and have more difficulty with accepting that their parents are becoming older. Based on the adaptation theory, adult children would adapt to the stress in life over time. Their perceived stress would decline thereafter as a result of adaptation process and then filial maturity was attained [22].

In contrast to prior studies, this study demonstrated that there was no correlation between primary caregiver role and perceived stress [15]. The stress and coping model indicates primary caregivers are associated with higher levels of perceived stress resulting from the impairments of care recipients and caregiver involvement $[17,24]$. Most prior studies examined the perceived stress of primary caregivers and confirmed primary caregivers were associated with high stress [25]. However, the general stress and caregiving-related stress were difficult to distinguish by only focusing on primary caregivers. Our study goes beyond the existing literature by comparing primary adult child caregivers with non-primary adult child caregivers, and found primary caregiver role was not significantly associated with perceived stress. It may be explained that the perceived stress of adult children mainly comes from other sides of their life, such as work and raising children, rather than taking care of their ageing parents. Additionally, high levels of familism would result in the appraisal of caregiving for ageing parents as less burdensome [11]. Chinese adult children have a strong sense of filial obligation and perceive taking care of ageing parents as a normal part of their life [26]. Previous research on Chinese immigrant sample also found providing caregiving for ageing parents can generate reward and mitigate burden for adult children $[27,28]$.

Our study found the overall health status between primary and non-primary adult child caregiver was not significant. Previous studies suggested primary caregivers were more likely to have poor physical health outcomes when compared with non-primary caregivers [25,29]. It is probably because those studies only focus on primary caregivers or based on non-representative samples that over-represent certain number of caregivers with poor health. Some studies reported negative effects of caregiving on physical health are most likely to be found in psychologically distressed dementia caregivers [21]. In addition, healthy caregiver hypothesis has been supported by prior studies on spouse caregivers, while our study extends existing understanding of healthy caregiver hypothesis by applying it to adult child caregivers.
Our study found adult children who were primary caregivers were more likely to have a job and have higher income. This is in contrast with prior studies that suggested competing roles of adult children create time constraints that detract from the ability to provide care and in particular, participation in the labor force appears to reduce caregiving to parents [29-31]. Some studies also found similar results that employment was not associated with primary caregiver role of adult children [32]. Based on our results, we proposed wealthy caregiver hypothesis to suggest the portrayal for primary caregiver would not always be in poor health with low income. However, this hypothesis needs to be tested in future research.

\section{Conclusions}

In sum, the present study identified adult children who were primary caregivers were more likely to have a job and have higher income. Primary caregiver role was not significantly associated with perceived stress. Health status was not significantly different between primary and non-primary adult child caregivers. As the number of ageing population largely increased in the past years, a more balanced and updated portrayal of family caregivers for older adults is needed to encourage more adult children to assume primary caregiver role and provide care for their ageing parents.

\section{References}

1. Pearlin LI, Mullan JT, Semple SJ, Skaff MM (1990) Caregiving and the stress process: an overview of concepts and their measures. Gerontologist 30: 583-594. [Crossref]

2. McCann JJ, Hebert LE, Bienias JL, Morris MC, Evans DA (2004) Predictors of beginning and ending caregiving during a 3 -year period in a biracial community population of older adults. Am J Public Health 94: 1800-1806. [Crossref]

3. Fredman L1, Doros G, Ensrud KE, Hochberg MC, Cauley JA (2009) Caregiving intensity and change in physical functioning over a 2-year period: results of the caregiver-study of osteoporotic fractures. Am J Epidemiol 170: 203-210. [Crossref]

4. Bertrand RM, Saczynski JS, Mezzacappa C, Hulse M, Ensrud K, et al. (2012) Caregiving and cognitive function in older women: evidence for the healthy caregiver hypothesis. J Aging Health 24: 48-66. [Crossref]

5. Brown SL, Smith DM, Schulz R, Kabeto MU, Ubel PA, et al. (2009) Caregiving behavior is associated with decreased mortality risk. Psychol Sci 20: 488-494. [Crossref]

6. Fredman L, Cauley JA, Hochberg M, Ensrud KE, Doros G (2010). Mortality Associated with Caregiving, General Stress, and Caregiving-Related Stress in Elderly Women: Results of Caregiver-Study of Osteoporotic Fractures. J Am Geriatr Soc 58: 937-943. [Crossref]

7. O'Reilly D, Connolly S, Rosato M, Patterson C (2008) Is caring associated with an increased risk of mortality? A longitudinal study. Soc Sci Med 67: 1282-1290. [Crossref]

8. National Alliance for Caregiving. Caregiving in the U.S. 2015.

9. Bastawrous M (2013) Caregiver burden--a critical discussion. Int J Nurs Stud 50: 431 441. [Crossref]

10. Lee Y, Smith L (2012) Qualitative research on Korean American dementia caregivers' perception of caregiving: Heterogeneity between spouse caregivers and child caregivers. Journal of Human Behavior in the Social Environment 22: 115-129.

11. Robinson Shurgot GS, Knight BG (2005) Preliminary study investigating acculturation, cultural values, and psychological distress in Latino caregivers of dementia patients. Hispanic Health Care International 3: 37-44.

12. Tang ST, Li C-Y, Liao YC (2007) Factors associated with depressive distress among Taiwanese family caregivers of cancer patients at the end of life. Palliative Medicine 21: 249-257. [Crossref]

13. Dong X, Chang ES, Wong E, Simon M (2012) The perceptions, social determinants, and negative health outcomes associated with depressive symptoms among US Chinese older adults. The Gerontologist 52: 650-663. [Crossref]

14. Dong X, Chen R, Simon MA (2014) Anxiety among community-dwelling U.S. Chinese older adults. J Gerontol A Biol Sci Med Sci. 69: S61-S67. [Crossref]

15. Wilks SE, Croom B (2008) Perceived stress and resilience in Alzheimer's disease caregivers: Testing moderation and mediation models of social support. Aging Ment Health. 12: 357-365. [Crossref] 
16. Lu N, Liu J, Lou VWQ (2015) Caring for frail elders with musculoskeletal conditions and family caregivers' subjective well-being: The role of multidimensional caregiver burden. Arch Gerontol Geriatr. 61: 411-418. [Crossref]

17. Lazarus RS, Folkman S (1984) Stress, appraisal, and coping. New York: Springer

18. Schwarz KA, Dunphy G (2003) An examination of perceived stress in family caregivers of older adults with heart failure. Experimental Aging Research 29: 221-235.

19. Cohen S, Kamarck T, Mermelstein R (1983) A global measure of perceived stress. $J$ Health Soc Behav 24: 385-396. [Crossref]

20. Zhang M, Simon MA, Dong X. 2014. The prevalence of perceived stress among US Chinese older adults. AIMS Medical Science 1: 40-56.

21. Pinquart M, Sörensen S (2007) Correlates of physical health of informal caregivers: a meta-analysis. J Gerontol B Psychol Sci Soc Sci 62: P126-137. [Crossref]

22. Merz E-M, Schulze H-J, Schuengel C (2010) Consequences of filial support for two generations: A narrative and quantitative review. Journal of Family Issues 31: 1530-1554.

23. Dong X, Zhang M (2016) The association between filial piety and perceived stress among Chinese older adults in greater Chicago area. $J$ Geriatr Palliat Care. 4. [Crossref]

24. Pinquart M, Sörensen S (2003) Associations of stressors and uplifts of caregiving with caregiver burden and depressive mood: a meta-analysis. J Gerontol B Psychol Sci Soc Sci 58: P112-128. [Crossref]
25. Schulz R, Sherwood PR (2008) Physical and mental health effects of family caregiving Am J Nurs 108: 23-27. [Crossref]

26. Funk LM, Chappell NL, Liu G (2013) Associations between filial responsibility and caregiver well-being: Are there differences by cultural group? Research on Aging 35: 78-95.

27. Lai DWL (2007) Cultural predictors of caregiving burden of Chinese-Canadian family caregivers. Can J Aging. 26: 133-147. [Crossref]

28. Lai DWL (2010) Filial piety, caregiving appraisal, and caregiving burden. Research on Aging 32: 200-223.

29. Pinquart M, Sörensen S (2003) Differences between caregivers and noncaregivers in psychological health and physical health: A meta-analysis. Psychol Aging. 18: 250267. [Crossref]

30. Fine MD (2012) Employment and informal care: Sustaining paid work and caregiving in community and home-based care. Ageing International 37: 57-68.

31. 31. Wang YN, Shyu YIL, Chen MC, Yang PS (2011) Reconciling work and family caregiving among adult-child family caregivers of older people with dementia: Effects on role strain and depressive symptoms. $J A d v$ Nurs. 67: 829-840. [Crossref]

32. Pillemer K, Suitor JJ (2014) Who provides care? A prospective study of caregiving among adult siblings. Gerontologist 54: 589-598. [Crossref]

Copyright: $@ 2017$ Mengting LI. This is an open-access article distributed under the terms of the Creative Commons Attribution License, which permits unrestricted use, distribution, and reproduction in any medium, provided the original author and source are credited. 